\title{
Norois
}

Environnement, aménagement, société

Conflits de lieux, lieux de conflits

\section{Quand les Calanques deviennent Parc National : disputes autour de la définition et de la répartition des efforts environnementaux et urbains induits}

When the Calanques become a national park: disputes surrounding the definition and allocation of induced urban and environmental efforts

\section{Cécilia Claeys, Arlette Hérat, Carole Barthélémy et Valérie Deldrève}

\section{OpenEdition \\ Journals}

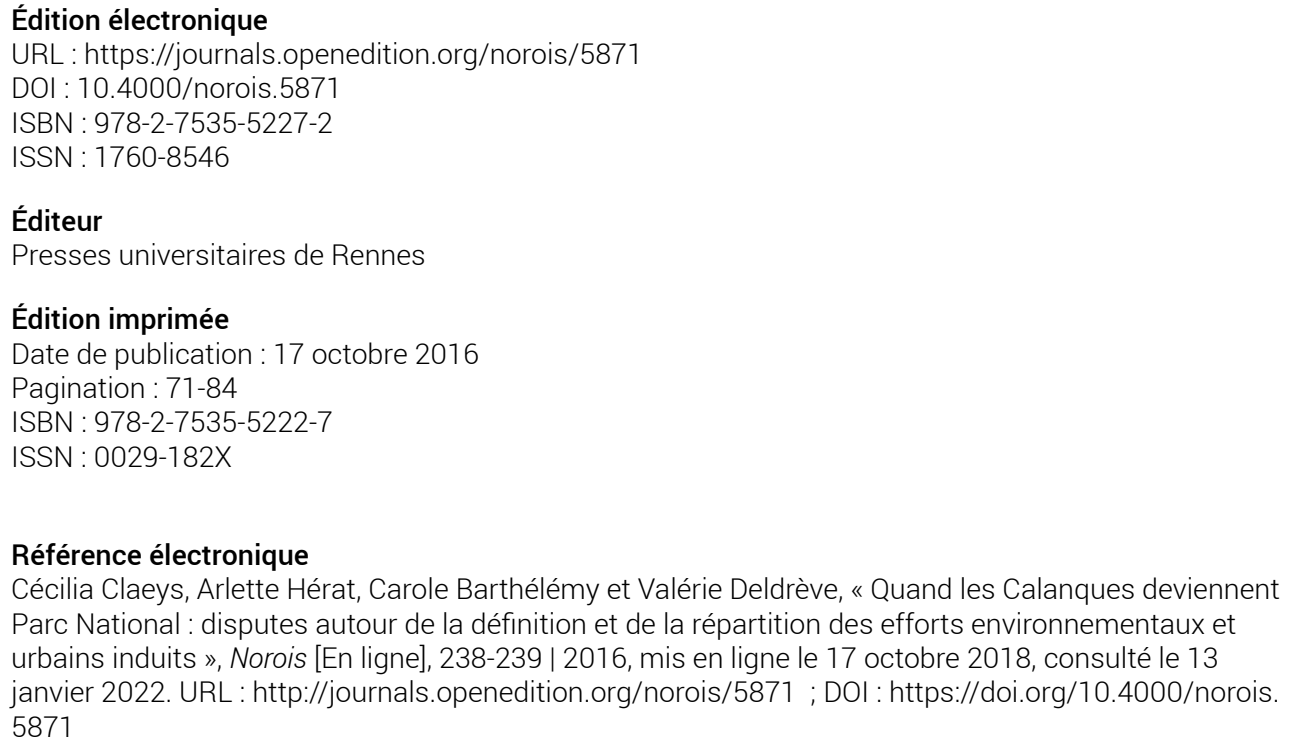

Référence électronique

Cécilia Claeys, Arlette Hérat, Carole Barthélémy et Valérie Deldrève, « Quand les Calanques deviennent Parc National : disputes autour de la définition et de la répartition des efforts environnementaux et urbains induits », Norois [En ligne], 238-239 | 2016, mis en ligne le 17 octobre 2018, consulté le 13 janvier 2022. URL : http://journals.openedition.org/norois/5871 ; DOI : https://doi.org/10.4000/norois. 5871 


Presses
Universilaires
de Rennes
www.pur-editions.fr
Revue en ligne : http://norois.revues.org

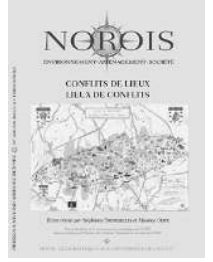

\title{
Quand les Calanques deviennent Parc National Disputes autour de la définition et de la répartition des efforts environnementaux et urbains induits
}

\author{
When the Calanques Become a National Park \\ Disputes Surrounding the Definition and Allocation \\ of Induced Urban and Environmental Efforts
}

\section{Cécilia Claeys ${ }^{a *}$, Arlette Hérat ${ }^{\mathrm{b}}$, Carole Barthélémy ${ }^{\mathrm{a}}$ et Valérie Deldrève ${ }^{\mathrm{c}}$}

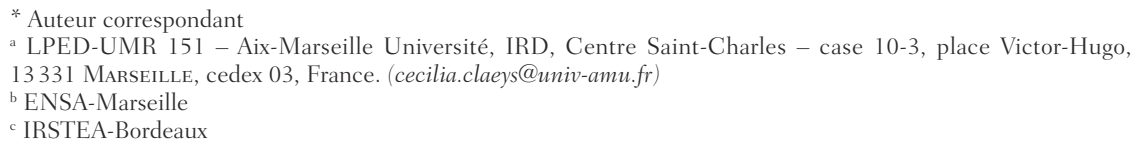

Résumé : Cet article propose une analyse des conflits relatifs à la création du Parc national des Calanques (18 avril 2012), premier parc périurbain européen. La grille de lecture proposée est celle de l'effort environnemental, défini comme la contribution, socialement différenciée et potentiellement inéquitable, des acteurs sociaux aux politiques publiques de protection de l'environnement. S'inscrivant dans le cadre d'analyse des inégalités environnementales, ce suivi sociologique initié en 2008, regroupe un dense corpus de données (observations directes, entretiens, questionnaires). L'analyse porte sur le processus de concertation et sur les premières implications de la requalification environnementale de ce lieu fréquenté de longue date par des populations urbaines locales. Une première partie porte sur les conflits relatifs aux modes de fréquentation du lieu et une seconde sur ceux relatifs à ses portes d'entrée. Mettant en regard ces deux dimensions, une dernière partie montre comment l'effort environnemental se double d'un autre type d'effort, que l'on qualifiera d'urbain.

\begin{abstract}
This article proposes an analysis of the conflicts surrounding the creation of the Calanques National Park, near Marseille (on April 18 ${ }^{\text {th }}$, 2012), the first periurban national park in Europe. Our approach has embraced the analytical framework of environmental effort. Environmental effort may be defined as the socially differentiated and potentially unfair contribution of social actors to public environmental-protection policies. As part of a wider environmental inequality framework, this sociological survey, initiated in 2008, has gathered a variety of data (direct observations, interviews, questionnaires). We shall look at both the importance of procedures implemented and the role these procedures play in the environmental reclassification of this area, which has long been used by local urban populations. The first part of the article focuses on conflicts relating to uses within the park itself, while the second focuses on conflicts concerning the management of its entry points. Linking these two dimensions, the final part of the article shows how environmental effort goes hand in hand with urban effort.
\end{abstract}

Mots clés: effort environnemental - inégalités environnementales - parcs nationaux périurbains - conflits d'usages - démocratie participative

Keywords: environmental effort - environmental inequalities - periurban - national parks - conflicts of use - participative democracy 


\section{INTRODUCTION}

La réforme juridique des parcs nationaux français a été pensée par le législateur comme une réponse aux conflits récurrents (Larrère et al., 2009, Laslaz, 2015) ralentissant (tel que les parcs de la Vanoise, des Pyrénées, de la mer d'Iroise...) ou empêchant la création de nouveaux parcs (Mont-Blanc, Clarée, Ariège, Corse...). Depuis le 14 avril 2006, le processus de création des nouveaux parcs nationaux répond, en théorie, aux principes de la démocratie participative et leur mode de gestion à ceux de la pluralité collégiale. En outre, il ne s'agirait plus d'extraire la nature des perturbations anthropiques (et réciproquement), mais de protéger des patrimoines naturels et culturels.

Officiellement né le 18 avril 2012, le Parc National des Calanques ( $\mathrm{PnCal}$ ) est le premier parc national français créé sous les auspices de ce nouveau cadre juridique. Une réussite pour le législateur qui serait ainsi parvenu à réinitialiser la dynamique de création des parcs. Néanmoins, l'apaisement des conflits par la participation et la collégialité n’a pas eu lieu. Au contraire, la création du PnCal peut être lue comme l'institutionnalisation de rapports de force contestés, aboutissant à une collégialité de l'entre soi (Deldrève et Hérat, 2012). En outre, la proximité, sinon l'enchevêtrement, entre urbanité et naturalité (Claeys et al., 2012) a complexifié et exacerbé les conflits locaux relatifs au statut et aux usages du PnCal (Guyot et al., 2014). Ce dernier est en effet mitoyen d'une métropole d'un million huitcent-mille résidents, dont Marseille seconde ville de France par son nombre d'habitants, marquée par sa pluriethnicité et ses fortes inégalités sociales et spatiales (Roncayolo, 1996; Donzel, 1998; Peraldi et al., 2015).

Cet article porte sur les conflits préfigurant la création du PnCal et accompagnant cette jeune structure en devenir. Ce travail s'inscrit dans l'analyse critique des conflits ${ }^{1}$ environnementaux (Charlier, 1999; Blatrix, 2000; Claeys-Mekdade, 2000 ; Godard, 2004; Mormont, 2006; Cadoret, 2006,

1. Les différences entre les notions de conflits ou de controverses relèvent en grande partie du cadre théorique dans lequel s'inscrivent les auteurs. La notion de conflit met l'accent sur les rapports de force en jeu, s'inscrivant dans une sociologie critique tandis que la notion de controverse est davantage mobilisée par la sociologie pragmatique mettant l'accent sur les processus dialogiques, les hybridations des savoirs (Callon et al., 2001), les formulations et les trajectoires des argumentaires (Chateauraynaud, 2011) notamment). Son apport spécifique est de développer une grille de lecture en termes d'effort environnemental ${ }^{2}$ qui se définit comme la contribution, socialement différenciée et potentiellement inéquitable, des acteurs sociaux aux politiques publiques de protection de l'environnement (Deldreve et Candau, 2014). Dans le cadre de la création et de la gestion d'un espace protégé, l'effort environnemental peut affecter les différents usagers, visiteurs d'un jour ou riverains, particuliers ou collectifs, professionnels, mais aussi les propriétaires fonciers privés ou publics. Cet effort environnemental peut prendre la forme de limitations d'accès, de régulations ou d'interdictions de certaines pratiques, de paiements de droits d'accès... La définition et l'attribution de cet effort environnemental, selon certains principes, donnent lieu à des tensions, voire à des conflits que cet article se propose d'analyser.

Après une présentation des cadres théorique et méthodologique, cet article déroulera l'analyse en trois temps. La première partie revient sur les formes de sélection sociale et de (dé/re)légitimation des usages induits par le processus de concertation. La seconde partie se focalise sur les conflits relatifs aux portes d'entrée du PnCal et les quartiers en cours de gentrification. La dernière partie, montre comment l'effort environnemental, subi ou consenti, pour protéger les Calanques dans le cadre de la politique du parc, se double d'un effort supplémentaire, qu'on qualifiera d'urbain, pour désigner la contribution inégalement supportée par les résidents dans le cadre des politiques de transformation urbaine. Cette contribution, justifiée au nom de la nécessité de la rénovation et de la densification urbaine, peut revêtir là encore des modalités diverses, comme la perte de son logement et d'un voisinage familier, de vue paysagère, d'espaces, et plus largement d'accès à certaines aménités.

\section{Cadres théorique ET MÉTHODOLOGIQUE}

La grille d'analyse proposée en termes d'effort environnemental s'inscrit dans une approche des politiques publiques, centrée sur la question des inéga-

\footnotetext{
2. La notion d'effort environnemental est parfois employée par des compagnies pour désigner ce qu'elles font afin de diminuer l'impact de leur production sur l'environnement. Mais l'acception et conceptualisation de l'effort environnemental, ici présentées, ont pour source l'élaboration du projet Effijie (ANR Socenv, 2014-2018, Candau et Deldrève coord.).
} 
lités environnementales. Ces dernières font figure de préoccupations croissantes dans les champs de l'action publique et de la recherche, aux États-Unis depuis les années 1980-90 sous l'influence du mouvement de l'Environmental Justice (Bullard, 2001; Taylor, 2000; Holifield, 2001) et en Europe depuis les années 2000 sous le registre du développement durable (Laigle, 2005; Faburel, 2010). Au-delà des controverses sur la terminologie à employer (inégalités écologiques ou environnementales), elles y sont définies, dans leur sens le plus extensif, comme des inégalités dans le rapport que les populations et groupes sociaux entretiennent avec leur environnement. Ces inégalités sont relatives à l'impact sur l'environnement que ces derniers ont de par leurs modes de production et de consommation. Elles ont également trait à leur exposition au risque, à l'accès et à la proximité aux aménités, capacité d'agir pour leur environnement et de bénéficier des politiques qui leur sont dédiées. Cumulatives, imbriquées à des formes d'inégalités économiques et culturelles, elles constituent de lourds enjeux de justice pour les politiques publiques et suscitent, de par le monde, nombre de résistances et de conflits locaux (Martinez-Alier, 2008). Il s'agit alors de savoir qui porte le plus préjudice à l'environnement et bénéficie de l'effort demandé pour le protéger et qui concrètement supporte cet effort. Les travaux d'économistes divergent dans leur réponse : pour certains les plus fortunés génèrent l'impact le plus important, mais aussi de par leurs ressources contribuent davantage aux mesures de protection (Lipietz, 1998); pour d'autres, ce sont les plus pauvres qui contribuent le plus, alors même que leur impact est moindre, tout comme le bénéfice qu'ils en retirent (Pye et al., 2008; Martinez-Alier, 2008). Les contestations locales de la politique des parcs nationaux (Larrère, 2009) et sa réforme de 2006 invitent à entrer dans ce débat controversé. Plus encore, parce qu'issu de cette réforme et situé en périphérie urbaine, le Pncal pose avec acuité la question de l'effort demandé aux résidents et aux usagers des Calanques.

À ce titre, les auteures mènent depuis 2008 un suivi sociologique de la création et de la mise en œuvre du PnCal. Entre 2008 et 2011, 32 observations des ateliers de concertation et mobilisations sociales (assemblées associatives, manifestations...) ont été réalisées et 115 entretiens auprès de gestion- naires et d'usagers participants et non participants à la concertation (Deldreve et Deboudt, 2012). Entre 2010 et 2012, des enquêtes qualitatives et quantitatives ont été réalisées auprès d'usagers marins et terrestres interrogés sur site. Les sites retenus, le mouillage de Sormiou, les îles du Frioul, le secteur de plongée des Pharillons (île Maïre), le littoral ouest du massif de Marseilleveyre, entre le Mont-Rose et la calanque de Marseilleveyre (figure 1), sont accessibles par les portes d'entrée marseillaises du territoire du parc. Ce corpus est constitué de 125 entretiens semi-directifs associés à une enquête par questionnaires auprès de 629 usagers, pilotée par Dominique Ami (Tatoni et al., 2013). Enfin, le programme Effijie est basé sur des approches qualitatives et quantitatives en cours de réalisation. Dans le cadre de cet article, un focus est proposé sur les premiers résultats issus des enquêtes qualitatives relatives à une zone d'interface entre des quartiers au sud de la ville de Marseille et le PnCal, allant de la Cayolle à la Madrague de Montredon (figure 1). Tandis que les deux premières campagnes d'enquêtes ont eu lieu durant les phases de concertation et de préparation du PnCal, ces enquêtes en cours sont pour leur part les témoins de la mise en place du parc national.

\section{QUI N'A PAS SA PLACE EN CE LIEU? DE L'INVISIBILITÉ À L'ILLÉGITIMITÉ DE CERTAINS USAGES DE LA NATURE}

La question de la fréquentation et les craintes de sur-fréquentation des Calanques furent au cœur des débats et des controverses qui ont accompagné la création du PnCal. Il s'agissait d'anticiper les effets d'appel que pouvait provoquer le parc sur de nouveaux usagers, mais aussi d'ordonnancer les usages préexistants à sa création. Néanmoins, les dispositifs de quantification de la fréquentation du PnCal sont à ce jour balbutiants. Dans les calanques, la pluralité des accès terrestres et marins, visibles ou invisibles, connus du grand public ou de quelques initiés complexifie l'exercice. En attendant la formalisation d'un protocole de suivi de fréquentation programmée pour 2016, le $\mathrm{PnCal}$ dispose principalement des comptages ${ }^{3}$ du nombre de visiteurs sensibilisés par les équipes

3. Initiés dès 2004 par le GIP-Calanques. 

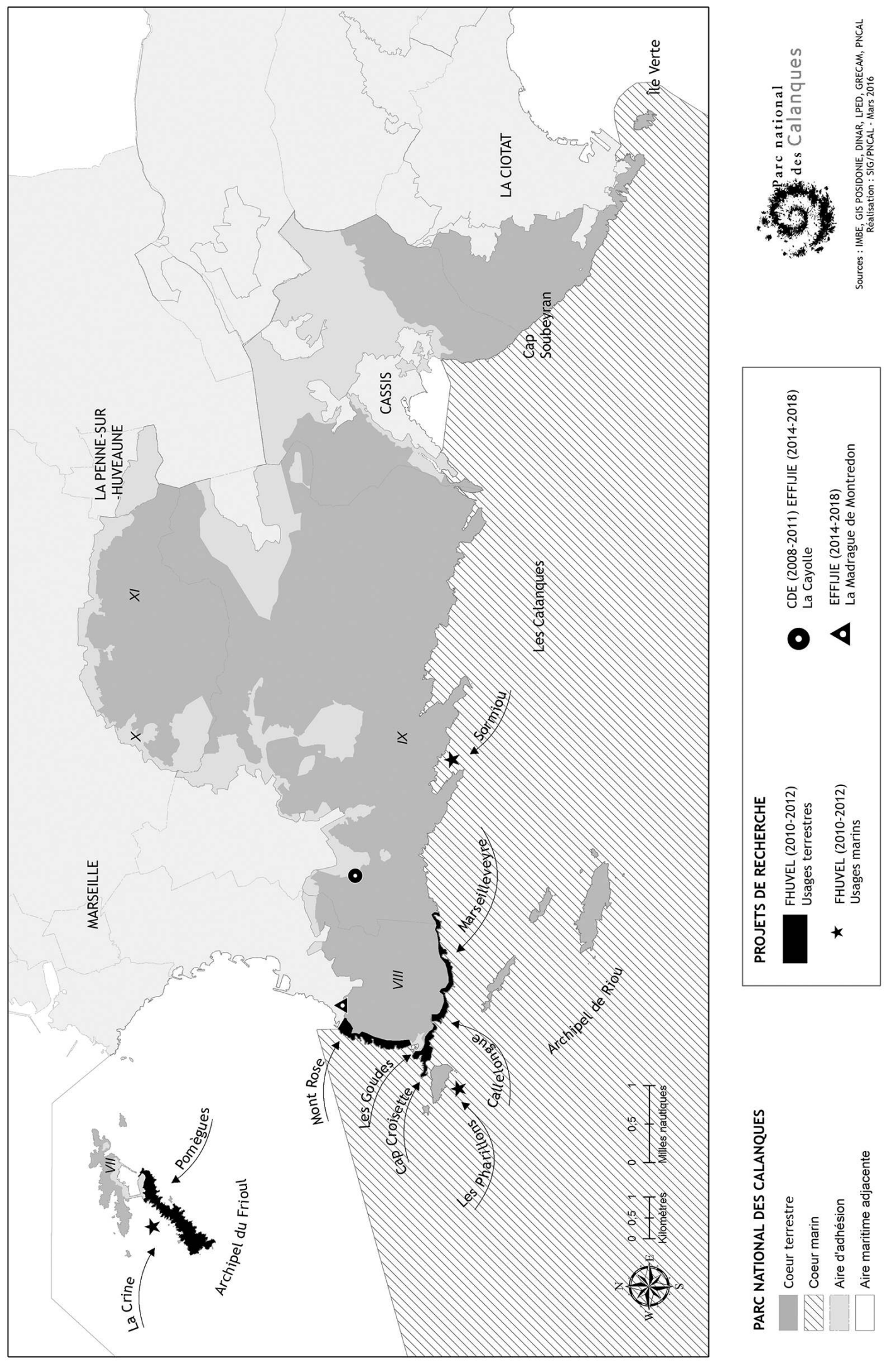

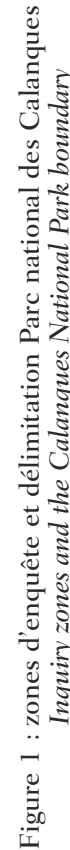


de saisonniers, équipes dont la taille varie d'une année à l'autre. Lors de la création du PnCal, ce manque de données chiffrées a été propice à une idéologisation des débats. L'augmentation de la fréquentation que pourrait induire la création du PnCal était un leitmotiv des réunions de concertation, chaque protagoniste priorisant des risques différents, en fonction de son statut et ses intérêts. Les représentants des usagers locaux tendaient à dénoncer prioritairement les risques d'un tourisme national et international croissant envahissant «leur terrain de jeu ancestral », tandis que les gestionnaires naturalistes s'inquiétaient en premier lieu des prélèvements et des impacts sur le milieu, quelle que soit l'origine géographique des contrevenants. Mais, par-delà ces différences, tous partageaient la même crainte vis-à-vis de la proximité d'une agglomération d'un million huit cent mille habitants. Paradoxalement, ce risque tend à être nuancé, sinon démenti, par les données produites par les gestionnaires eux-mêmes. En effet, quel que soit le nombre de visiteurs comptabilisés d'une année sur l'autre par les saisonniers ${ }^{4}$, la part de Marseillais y demeure stable, se situant entre $19 \%$ et $37 \%$ selon les types d'usagers (marins ou terrestres) (PnCal, 2014). Parce que réalisées auprès d'usagers interrogés dans plusieurs sites proches de la ville de Marseille, nos enquêtes qualitatives et quantitatives in situ donnent à voir, pour leur part, une plus grande proportion de Marseillais. Cependant, même aux alentours des accès marins et terrestres les plus proches de la ville, les Marseillais ne sont pas majoritaires parmi les personnes interrogées. En effet, 46,71\% des répondants au questionnaire réalisé sur site en 2012 dans le cadre du programme FHUVEL (Tatoni et al., 2013) habitent Marseille, 9,87\% habitent dans les Bouches-du-Rhône (BDR) hors Marseille et 3,07\% en région Provence-Alpes Côte d'Azur, hors BDR. En outre, parmi les Marseillais interrogés, $44 \%$ habitent dans les $8^{\mathrm{e}}$ ou $9^{\mathrm{e}}$ arrondissements adossés au massif des calanques. Tandis que les arrondissements populaires plus éloignés du territoire du PnCal $\left(2^{\mathrm{e}}, 3^{\mathrm{e}}, 14^{\mathrm{e}}, 15^{\mathrm{e}}\right)$ sont sous représentés. Enfin, les enquêtes qualitatives (entretiens et observations directes) révèlent des phénomènes micro-scalaires difficilement quantifiables.

4. Le nombre de visiteurs sensibilisés dépend de la taille des équipes de saisonniers qui peut varier d'une année sur l'autre.
La population marseillaise a certes investi de longue date le massif des Calanques comme espace de prélèvement, de ressourcement et de loisirs (Massena-Gourc, 1994). En conclure pour autant que les Calanques seraient exposées à l'assaut des populations marseillaises procède d'un fantasme socialement construit. À ce titre, ces craintes des masses urbaines ne portent pas seulement sur leur nombre, mais également sur leur «qualité ». Les peurs de sur-fréquentation glissent dès lors vers une stigmatisation de la «mal-fréquentation » (Deldrève et Hérat, 2012). L'analyse des débats menant à la création du PnCal rend compte de processus de sélection et de normalisation amenant à valoriser certains usages et à en discréditer d'autres. En privilégiant la tenue de réunions thématiques sur invitation, l'organisation même de la concertation a favorisé ces processus de sélection. En invitant des représentants associatifs, professionnels, institutionnels, le GIP-Calanque en charge de l'organisation de la concertation a favorisé la parole de groupes structurés, renforçant l'invisibilité sociopolitique des catégories d'usagers non structurées. Les participants les plus loquaces et les plus influents pouvaient en outre représenter des catégories d'usagers ayant un poids démographique modeste (comme les cabanoniers et les chasseurs) (Deldreve et Deboudt, 2012). Les connivences culturelles, les lobbyings et les négociations « notabiliaires » ont contribué à la reconnaissance réglementaire de certains usages au détriment d'autres, non sans quelques paradoxes. Par exemple, les chiens en liberté sont interdits, sauf pour les chasseurs, de même les feux sont prohibés, mais les barbecues des cabanonniers sont tolérés. Ces processus de sélection tendent à délégitimer les pratiques populaires contemporaines au profit d'un «bon usage de la nature » correspondant in fine aux usages caractéristiques des couches moyennes et supérieures, érigés en tradition et appréhendés comme l'alternative positive à la fréquentation de masse (Ginelli et al., 2014).

Objet de débat, enjeu social, culturel et politique, la sur-fréquentation des Calanques relève du postulat idéologique, mais demeure un constat empirique difficilement objectivable. Sur le terrain, les usagers « ordinaires » en ont pour leur part une vision plus nuancée, comme le révèlent les entretiens semi-directifs menés in situ. Sur terre comme en mer, les usagers rencontrés s'accordent certes sur 
l'idée que les Calanques sont très fréquentées. Ce constat n'est cependant pas exprimé sur le registre de la dénonciation. Même les usagers déclarant une aversion pour la grégarité demeurent nuancés. Pragmatiques, ils optent pour des stratégies d'évitements spatio-temporels, choisissant des lieux reculés plus difficilement accessibles et/ou l'arrière saison. Les critiques de la fréquentation recueillies sur le terrain sont qualitatives bien plus que quantitatives. Elles rejoignent en cela les dénonciations d'une mal-fréquentation formulées lors des réunions de concertation. Sur le terrain, cette mal-fréquentation tend à être attribuée aux " autres ", chaque type d'usagers pouvant se trouver tour à tour accusé ou accusateur.

La tolérance des usagers rencontrés vis-à-vis de la forte fréquentation des Calanques appartient à deux registres différents, mais non contradictoires. En premier lieu, une conception égalitaire de l'accès à la nature tend à être défendue, même par les usagers les plus critiques, tel ce plaisancier : «Ben oui en tant que local on va dire qu'elles [les Calanques] sont trop fréquentées bien sûr, bien sûr. Mais bon on ne peut pas interdire aux gens de voir ce qui est beau. Nous on fait pareil quand on va visiter des endroits merveilleux dans le monde. Donc c'est vrai qu'on peut difficilement interdire aux gens de venir voir les Calanques, mais il y a de plus en plus de monde." Ce propos égalitariste peut aussi procéder d'un déni stratégique par crainte d'un renforcement de la réglementation (Claeys, 2014). Qu'il soit « sincère » ou stratégique, l'argumentaire de ces usagers relève néanmoins des principes de démocratisation de l'accès à la nature et de justice environnementale, exprimant unanimement la crainte d'un renforcement de la réglementation induit par la création du PnCal. Par exemple, au moment où la concertation bat son plein, ces pêcheurs à pied rencontrés sur le rivage s'inquiètent : "On est là pour prendre quatre poissons, on dépeuplera jamais la mer, je vous le dis [...] On va nous l'interdire et là ça va... Vous allez voir que ça va être dur là. »

Les oppositions au renforcement réglementaire sont exacerbées par un sentiment d'injustice. Les usagers rencontrés rechignent d'autant plus à accepter l'effort environnemental que leur demande le PnCal qu'ils considèrent leur impact sur le milieu sans commune mesure avec d'autres sources de perturbation et de pollution. Les rejets de la station d'épuration de la ville de Marseille dans la Calanque de Cortiou et ceux des boues rouges industrielles dans la rade de Cassis sont tout particulièrement pointés du doigt (figure 2). Les propos les plus vindicatifs ont été recueillis auprès des usagers de la mer : plaisanciers, plongeurs et pêcheurs. Par exemple, ce couple de plaisanciers s'insurge «À partir de là, je trouve complètement anormal qu'il y ait des rejets comme ça et alors on crée un parc naturel, tu vois. On a l'impression qu'on renvoie... le problème sur d'autres, quoi. Sur le peuple. Alors qu'en fait, le peuple, lui, il a besoin de ses Calanques, parce que c'est ses Calanques à lui... C'est la liberté... Et tu vois, c'est son oxygène... Liberté de circuler...»

En soumettant certains acteurs sociaux plus que d'autres à des demandes d'effort environnemental, la création du PnCal tend à susciter des sentiments d'injustice chez certains usagers. De ce point de vue, l'(in)acceptation d'un effort environnemental est directement inféodée au sentiment de justice ou d'injustice qu'il suscite. Ce sentiment d'injustice tend à être renforcé dans le cadre d'un parc national périurbain du fait de la proximité spatiale entre ville et espace protégé. Il est d'autant plus frustrant de renoncer à sa façon de fréquenter un espace que ce dernier est proche et familier, et plus encore lorsque cet espace endosse des statuts pluriels, voire ambigus, tour à tour, ou bien tout à la fois appréhendé comme nature remarquable et ordinaire, comme nature sauvage et domestique (Claeys et al., 2012). Les entretiens réalisés auprès de ces usagers urbains donnent à voir une association des registres lexicaux du magnifique (les expressions «beauté », « joli », «splendide ", «magnifique » sont récurrentes) et du familier ("C'est mon jardin que j'aime, que j'aime bien fréquenter, un jardin » résume par exemple un plaisancier local fréquentant de longue date les îles du Frioul).

Dès la création du PnCal, la volonté de réglementation des flux d'usagers a amené les gestionnaires à porter une attention toute particulière aux portes d'entrée urbaines, élargissant les enjeux d'effort environnemental aux riverains, qu'ils soient ou non usagers des Calanques. 


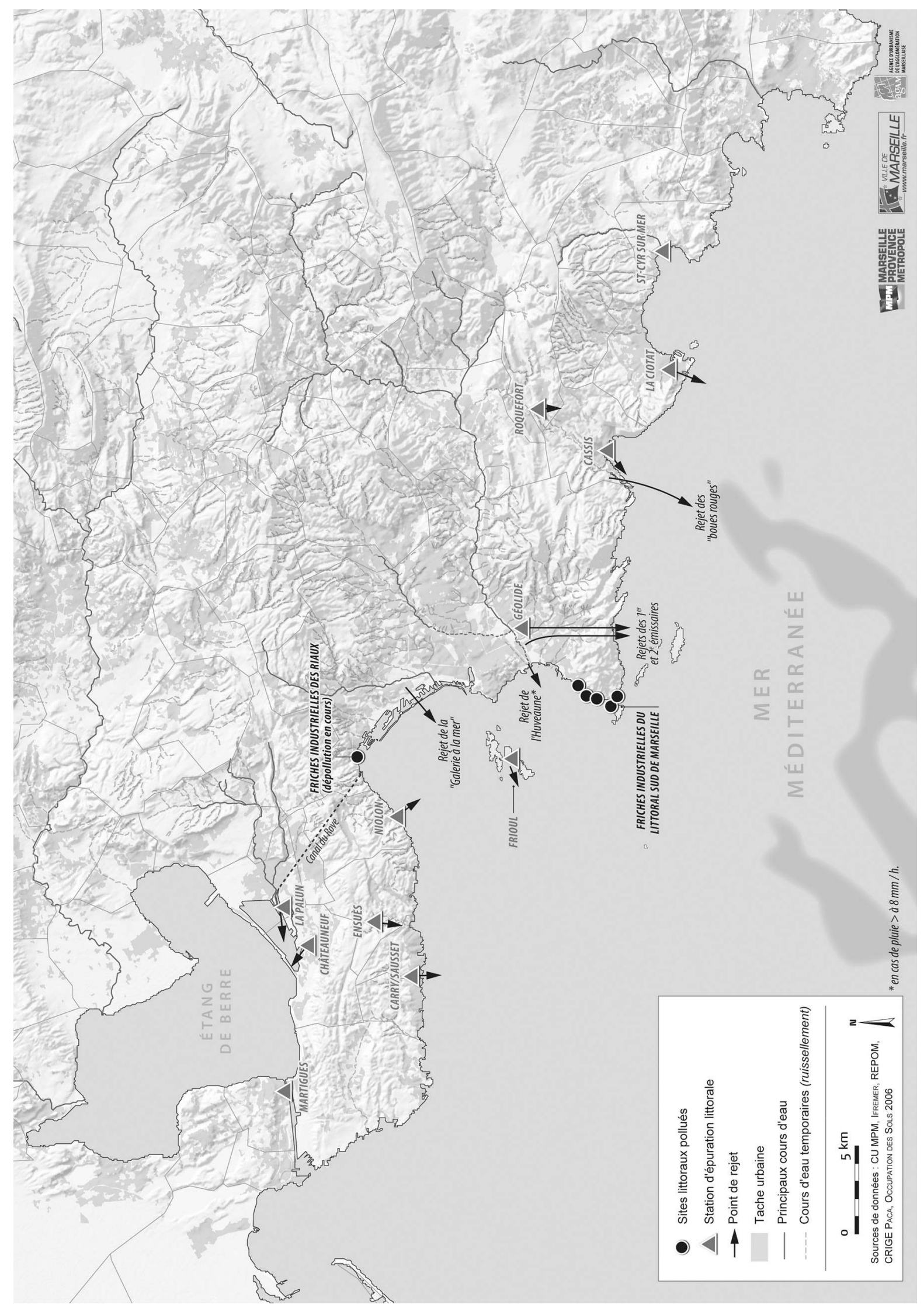

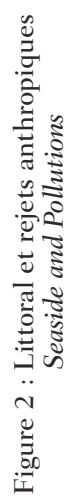




\section{«Conflits de voisinage »: QUAND DES MARGES URBAINES DEVIENNENT PORTES D'ENTRÉE D'UN PARC NATIONAL}

Les quartiers sud de Marseille sont présentés de manière courante comme étant les quartiers bourgeois de la ville, caractéristique dont rend bien compte une batterie d'indicateurs basée sur des moyennes (professions et catégories sociales, valeurs foncières et immobilières, revenus...). Cependant, compte tenu de leur histoire comme de la géographie de la ville, ces quartiers révèlent aussi de grandes disparités micro-scalaires rendant compte d'une mosaïque plus complexe. L'urbanisation centrifuge de la ville (présente dans les traces des 101 villages, ou dans les représentations des milliers de bastides ${ }^{5}$ disséminées dans un vaste territoire) s'est accommodée d'une faiblesse de la planification, tout en s'appuyant sur les dessertes offertes par les infrastructures publiques (canal, chemin de fer, réseau de tramway). Les pieds de colline du massif des Calanques comme les bords du littoral sud deviennent ainsi le réceptacle d'activités industrielles (Daumalin, 2013), de cabanons pour les loisirs, d'habitat des catégories populaires dont une partie est réalisée sous forme d'auto-construction, dispositif encouragé au début du $\mathrm{xx}^{\mathrm{e}}$ siècle par le pouvoir municipal comme parade aux problèmes d'insalubrité du centre-ville (Gontier, 1991). En y localisant des installations publiques dont la mise à distance de la ville est souhaitée (telles que prison, camps...), les édiles contribuent à fabriquer des marges urbaines caractérisées par leur éloignement du centre ville (à plus de $10 \mathrm{~km}$ du Vieux Port) et un défaut d'équipement (figure 3).

À l'échelle de la ville, la division sociale du territoire marseillais entre un nord populaire et un sud bourgeois s'affirme à partir de 1880 avec l'extension du Port de commerce et se renforce après la Seconde Guerre mondiale avec la programmation de vastes programmes de logements sociaux. Les quartiers Sud bénéficiant des aménités d'un littoral balnéaire et des collines voient dans le dernier quart du $\mathrm{Xx}^{\mathrm{e}}$ siècle les discontinuités urbaines s'amoindrir progressivement (les terres de maraîchage s'urba-

5. Comme celle de Pierre Chevallier de Soissons, « Plan du terroir, ville, port et rade de Marseille et ses environs ", début du XVIII ${ }^{e}$ siècle représentant « la plus grande ville du monde». nisent sous la forme de grandes opérations d'aménagement) et l'arrivée d'une nouvelle économie fondée sur le tertiaire, les centres commerciaux et le tourisme en remplacement de l'industrie. La création du PnCal en 2012 (à la suite de la création du site classé ${ }^{6}$ dans les années soixante-dix), peut ainsi apparaître comme parachevant une dualité du territoire marseillais préexistante (Donzel, 1998; 2014).

La Cayolle et la Madrague de Montredon, en confrontation directe avec le cœur de parc, situés dans deux des arrondissements les plus aisés de Marseille $\left(\mathrm{VIII}^{\mathrm{e}}\right.$ et $\left.\mathrm{IX}^{\mathrm{e}}\right)$, témoignent de ce passé populaire et d'un processus de gentrification en cours (Jourdan 2008) ${ }^{7}$. Notre propos questionne, du point de vue des décideurs locaux et des résidents, le passage d'une situation de marge urbaine - qu'il faut cependant relativiser selon les cas, compte tenu de la desserte en tramway du village de la Madrague de Montredon au début du $\mathrm{Xx}^{\mathrm{e}}$ siècle - à celle de porte d'un parc national.

En ce qui concerne le territoire marseillais, le décret de prise en considération du PnCal présentait un périmètre de cœur de parc calqué sur le site classé de 1975 et une aire optimale d'adhésion réduite à une portion congrue, du fait d'une conception de l'aire d'adhésion fondée sur des qualités environnementales excluant les zones urbanisées à l'exception du campus universitaire de Luminy. Ce n'est qu’à la suite des nombreuses réserves émises par le rapporteur de la commission d'enquête publique en vue de la création du PnCal qu'une partie du quartier de la Cayolle, objet d'une opération de renouvellement urbain, est inscrite en aire d'adhésion. Pour autant ce même rapport n'avait pas conclu à l'intérêt d'intégrer en aire d'adhésion le site de l'ancienne usine Legré Mante (ayant fermé ses portes en 2009) malgré les demandes exprimées de riverains de la Madrague de Montredon, doublement inquiets des conditions de circulation déjà saturées en période estivale et d'un projet immobilier sur la friche polluée. Ces constats renvoient au refus de la mise en débat public, lors des réunions de concertation sur la charte du PnCal, des questions régissant l'interface entre le système urbain et l'espace protégé (Deldrève et Hérat, 2012), contredisant ainsi la

\footnotetext{
6. Au titre de la loi du 2 mai 1930 sur la protection des monuments naturels et des sites de caractéristique artistique, historique, scientifique, légendaire ou pittoresque.

7. Pour des éléments plus précis sur les deux contextes étudiés, voir Hérat, 2012 et Barthélémy et Hérat, 2016.
} 
volonté de créer « le premier parc national périurbain d'Europe » mise en avant par les acteurs ayant en charge sa création. Ainsi les réflexions et projets sur l'accessibilité du PnCal (canalisation des flux touristiques, nature des modes de déplacements) ou la qualité des transitions paysagères entre ville et espace naturel ont été travaillées parallèlement par l'agence d'urbanisme de l'agglomération marseillaise (AGAM) et les services municipaux. Si la stratégie municipale ${ }^{8}$ vise à densifier le nombre de logements aux portes du parc (plus 300 logements environ pour chacun de ces secteurs dans les premières esquisses de projet), et ce malgré les fortes contraintes de desserte des secteurs de confins auxquelles s'ajoute le report récurrent de la réalisation du Boulevard Urbain Sud et des transports publics associés, elle révèle cependant une différence dans l'approche des deux quartiers.

Selon le Schéma de Cohérence Territoriale de Marseille-Provence-Métropole, dont le Document d'orientation générale a été approuvé en juin 2012, la Madrague de Montredon est clairement identifiée comme une porte majeure du PnCal (figure 3) et pour répondre à la congestion de la voie d'accès terrestre, des navettes maritimes viennent d'être mises en place pendant la période estivale entre le port de la Pointe Rouge et la calanque des Goudes. Ce rôle entérine la mutation résidentielle et touristique du littoral sud conduisant au renforcement des catégories moyennes et supérieures et effaçant peu à peu les traces d'une histoire industrielle et populaire.

Pour sa part, le quartier de la Cayolle n'a pas été retenu comme porte principale d'accès au Parc national, alors qu'il bénéficie d'une opération publique d'envergure avec le projet mené dans le cadre de l'ANRU et qu'il permet un accès direct à la calanque de Sormiou (une des icônes du parc avec la présence de cabanons, d'une plage et d'un petit port). Nous y voyons une stratégie temporaire, prenant acte de la marginalité sociale d'un secteur et liant sa mise en visibilité à une mutation sociale de population. Cette mutation paraît être l'enjeu sousjacent du projet de l'ANRU à la Cayolle (Hérat, 2012). En ciblant les quelques démolitions sur les cités de promotion familiale (figure 4) édifiées dans

8. Stratégie révélée par l'analyse du Plan local d'urbanisme (mis en révision en février 2009 et approuvé en juin 2013), de la convention de renouvellement urbain avec l'ANRU sur le secteur de la Cayolle (signée en octobre 2011) et de l'arrêté du permis de construire sur le site de Legré Mante (novembre 2011).

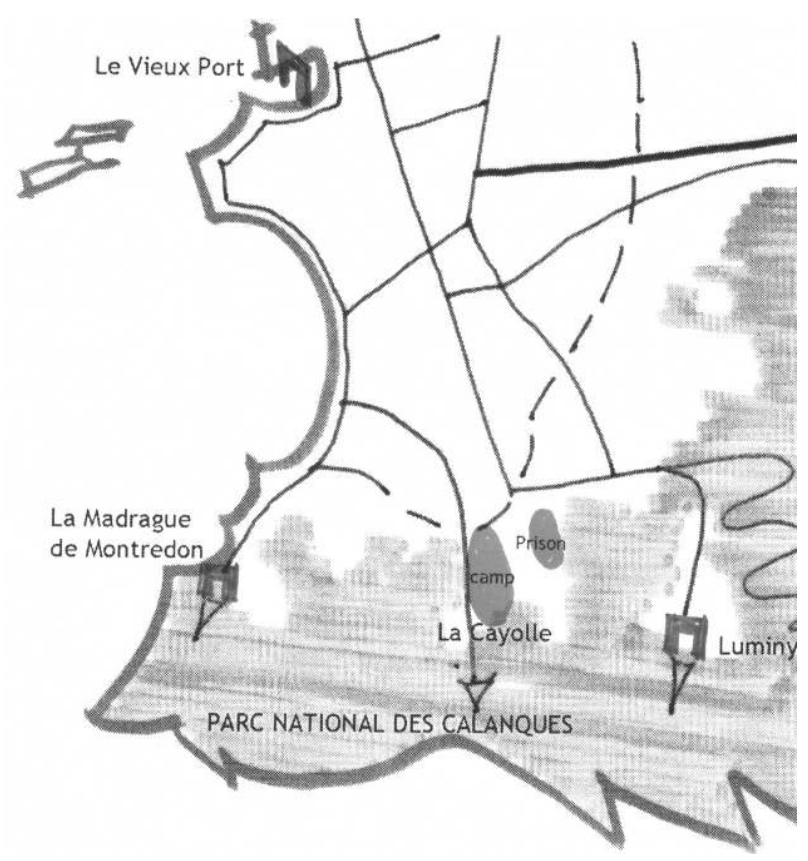

Figure 3 : Les portes principales du parc (Arlette Hérat) Main entrance of the parc

les années quatre-vingt en vue de résorber le bidonville ayant pris place à la suite du camp de transit (Témime et Deguigné, 2001), et en ré-agençant les espaces selon le principe de "résidentialisation ${ }^{9}$ », le plan de réaménagement du quartier bouleverse des usages populaires tissés autour de relations intimes (et ancrées dans les mémoires) entre habitants des cités et colline.

\section{DE L'EFFORT ENVIRONNEMENTAL À L'EFFORT URBAIN}

Les nouvelles réglementations issues du PnCal, que l'on peut associer à un effort environnemental demandé aux usagers du site, se doublent d'«un effort urbain » pour les usagers qui sont également habitants des marges. Cet effort urbain prend plusieurs formes.

Tout d'abord, la constitution du PnCal, en maintenant une dichotomie forte entre les espaces naturels protégés et la ville, fait reposer une forme de « pression » sur les franges urbaines, lieux straté-

9. La résidentialisation, principe essentiel des opérations de renouvellement urbain, est basée sur la volonté de fragmenter les espaces ouverts qui caractérisent les ensembles modernes. Ce principe conduit à clarifier les domanialités et à définir des échelles intermédiaires de groupement de logements. Il s'accompagne le plus souvent de fermetures résidentielles. 


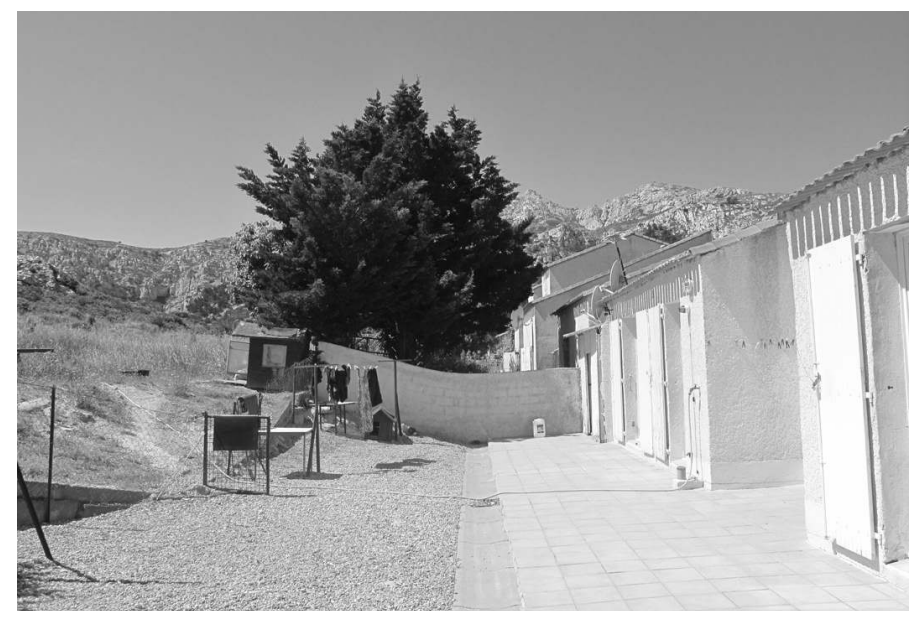

Figure 4 : Logements La Cayolle (photo Arlette Hérat) Housing La Cayolle

giques qu'il faudrait maîtriser. Maîtriser tout d'abord la circulation, notamment pendant les week-ends et les périodes de vacances. Un argument récurrent des habitants qui étaient opposés au parc avant sa création était l'augmentation de la circulation, contribuant à renforcer une gêne déjà bien installée. Nous avons ainsi recueilli le témoignage d'une habitante de la Madrague qui racontait comment, déjà, lorsque le tramway permettait d'arriver jusqu'aux Calanques, le trafic était saturé le dimanche. La présence du PnCal pouvait ainsi générer une augmentation de la nuisance liée aux transports, ce qui reste encore à vérifier. Un nouveau plan de circulation est fortement décrié et constitue un des arguments principaux d'une mobilisation locale réunissant des associations de résidents et de militants environnementalistes.

La maîtrise des marges est également stratégique sur le plan du foncier. Une étude préliminaire réalisée sur l'offre immobilière dans certains quartiers Sud, proche des portes des Calanques, a montré une dynamique commune et ancienne d'offres d'ensembles résidentiels majoritairement destinés aux individus des classes moyennes et supérieures, s'accommodant de la proximité de zones d'habitat populaire. Si les intitulés des résidences empruntent à la proximité du $\mathrm{PnCal}$ («Bleu Calanques », « Cap Morgiou »), les aménités mises en exergue portent plus sur l'accès rapide de ces quartiers à l'autoroute qu’à celle du massif des Calanques. L'étude du passage du Plan d'Occupation du Sol (POS) au Plan Local d'Urbanisme (PLU) a également montré que la présence du PnCal n'avait pas contribué à renforcer la réglementation dans les marges. Alors que le POS prévoyait une bande de 50 mètres de large en limite avec le massif, créant une "zone tampon » où l'urbanisation était réglementée, le PLU y substitue une " orientation d'aménagement thématique sur les franges urbaines » sans valeur prescriptive. L'ancienne zone tampon se trouve ainsi découpée selon des règlements divers, tels que «maîtrise forte de l'urbanisation, franges ville-nature », «Tissus discontinus de types collectifs denses et/ou à densifier » autorisant l'habitat collectif jusqu'à 16 m de hauteur. À ce jour, la présence du PnCal ne semble pas directement infléchir les dynamiques immobilières des quartiers Sud de Marseille. Celles-ci se caractérisent par la densification des franges urbaines, processus récent dont il est encore difficile de mesurer les conséquences en termes d'effort urbain que devront assumer les habitants (figure 5).

Ceux-ci cependant en perçoivent déjà la menace. C'est ainsi qu'un projet immobilier portant sur le site de l'ancienne usine Legré-Mante suscite une forte mobilisation à son encontre, alliant collectifs d'habitants et associations des secteurs de l'environnement et la santé. Ce site fortement impacté en matière de contamination des sols par les métaux lourds tels que plomb, arsenic, cadmium, antimoine, mercure, se distingue dans le massif des Calanques par la longévité des activités qui s’y sont maintenues pendant plus de deux siècles. Alors que les autres friches du massif ont été déclarées « sites orphelins », conduisant l'État à prendre en charge les questions de leur mise en sécurité ${ }^{10}$, ici c'est au propriétaire - suite à la défaillance du dernier exploitant - qu'échoit cette responsabilité. C'est ainsi que le projet de dépollution du site est intégré dans un projet immobilier. À la suite de recours judicaires engagés par deux associations de quartier, en novembre 2013, un Comité de liaison pour la défense de la santé des riverains du site LegréMante se constitue dans le but de rassembler largement la population «face à un projet dont les conséquences sanitaires ne sont toujours pas mesurées, en exigeant des pouvoirs publics (Préfecture et Mairie de Marseille) une ÉTUde D’ImPACT sanitaire

\footnotetext{
10. d'après la circulaire du 26 mai 2011 relative à la cessation d'activités d'une Installation Classée - Chaîne de responsabilités - Défaillance des responsables.
} 

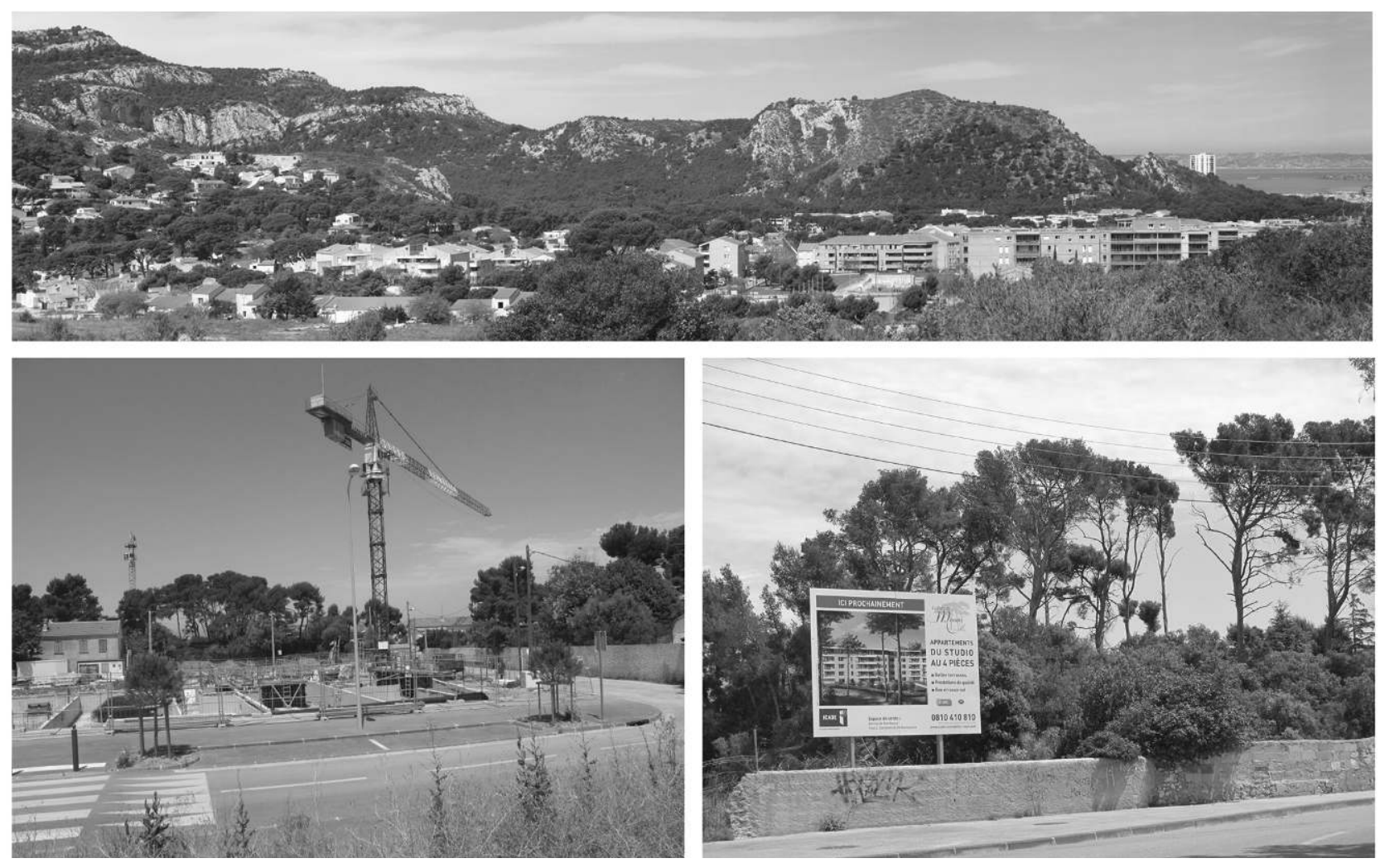

Figure 5 : L'urbanisation des franges (photos Arlette Hérat)

En haut : le quartier de la Cayolle où 300 logements supplémentaires sont programmés. En bas : programmes immobiliers en cours de chantier ou annoncé.

Urbanization fringes

Up : Cayolle neighborhood where 300 additional housing units are planned. Bottom : housing programs in progress or announced.

et une ENQUÊTE PUBLIQUE » (Bulletin d'information $\mathrm{n}^{\circ} 1$ - janvier 2014) ${ }^{11}$.

Des observations régulières lors des réunions du Comité permettent d'appréhender comment cette opposition au projet s'est constituée, oscillant entre divers arguments (risques sanitaires, pression urbaine et circulation), mais adoptant une stratégie focalisée uniquement sur la défense de la santé publique : "Ils voudraient nous faire dire que c'est une croisade anti-promoteur, ce n'est pas ça»se défend le coordinateur du Comité, lors de la réunion du 15 avril 2014. L'accent est mis sur la différence de traitement de la part des pouvoirs publics entre les différents sites pollués du secteur : "Ils [les scientifiques] disent qu'il ne faut rien toucher entre l'Escalette et Callelongue, et sur Legré-Mante on [les services de l'État] dit qu'on peut remuer $90000 \mathrm{~m}^{3}$ de terre! » souligne ce même coordinateur, lors de la

11. Pour des informations plus précises voir le site internet du Comité de Santé du Littoral Sud : [http://wwww.comite-sante-littoral-sud.org]. réunion du 8 octobre 2015. Bien que la majorité des participants partage de nombreuses craintes face à l'effort urbain qui se profile, la stratégie d'action du Comité arrive jusqu'à présent à combiner un élargissement de l'ancrage auprès des associations locales avec une mobilisation centrée sur la défense de la santé $^{12}$ : «Ce n'est pas le moment pour discuter de l'immobilier, on n'est ni pour, ni contre », rappelle ce représentant d'association environnementale, lors de la réunion du 28 octobre 2014. Pour autant, les préoccupations liées au cadre de vie ne sont pas complètement exclues, comme en témoignent la participation aux manifestations liées à la circulation, au nettoyage des Calanques ou le versement aux dossiers d'enquête publique en vue de la réalisation du Boulevard Urbain Sud et de la modifica-

12. Traduite dans la demande de création, à l'initiative de quinze associations dont huit CIQ, d'une Commission de Suivi de Site (CSS). La CSS est une structure d'information et de concertation obligatoirement mise en place sur des installations classées pour la protection de l'environnement (ICPE), selon la circulaire du 15 novembre 2012 relative à la mise en application du décret n $\mathrm{n}^{\circ}$ 2012-189 du 7 février 2012. 
tion du PLU (dernier trimestre 2015). Dans cette situation de conflit, les relations avec le PnCal sont, jusqu'à présent, empreintes de défiance, cette dernière étant renforcée depuis l'autorisation donnée à la poursuite des rejets industriels (boues rouges) en cœur marin. Si le PnCal n'apparait pas aujourd'hui comme un médiateur possible pour le Comité, la situation du quartier en tant que porte de parc national semble un argument pour en promouvoir la défense de sa qualité paysagère.

La pression immobilière peut également prendre une autre forme dans l'achat et la rénovation d'habitats populaires. Nous avons ainsi rencontré un jeune habitant de la Madrague qui nous expliquait comment il s'était senti harcelé par un agent immobilier qui voulait « lui faire » vendre la maison de ses parents. Ce témoignage rend compte du dernier élément qui constitue, l'effort urbain. Celui-ci repose sur les liens étroits qui unissent les habitants des marges aux milieux naturels adjacents. Nos enquêtes convergent pour montrer que les pratiques de balade, de cueillette, de chasse ou de pêche sur le littoral constituent un élément central dans le fait d'habiter ces quartiers. Le cadre de vie, entre mer, colline et « campagne » selon une habitante est largement revendiqué comme une aménité également pour les individus de milieux sociaux populaires (essentiellement composés d'ouvriers et d'employés) ${ }^{13}$. Pour ces habitants, il n'y a pas de distinction entre la ville et la nature, leur lieu de vie, les collines et la mer. Cette imbrication, sinon cette hybridation, est largement sous-estimée par les acteurs institutionnels qui insistent, au contraire, sur la distinction entre la ville et les milieux naturels. L'effort urbain résulte de cette segmentation institutionnalisée qui, en réglementant l'accès aux milieux naturels et leurs usages, peut conduire à remettre en cause leur cadre de vie.

Concernant la cueillette, le parc autorise le prélèvement de quelques espèces dans certaines quantités (l'asperge sauvage, le thym, le romarin et le fenouil) pour une « consommation individuelle ou familiale ", c'est-à-dire, concrètement, en limitant leur cueillette à un «bouquet ", de façon à ce que l'index et le pouce puissent se toucher. Si cette réglementation semble peu contraignante, des témoignages d'habitants contrôlés par les gardes

13. Un tel processus a été précédemment observé sur d'autres territoires, par exemple sur la côte d'Opale (Deldreve, 2011). rendent compte d'un profond malaise à l'égard de cette surveillance. Quant à la pêche, les zones de non prélèvement en milieu marin obligent les pêcheurs à se déplacer hors de leurs sites habituels. D'autres riverains insistent au contraire sur le flou des interdictions qui ont régulièrement été modifiées, profitant en quelque sorte à la continuité des pratiques existantes.

En ces lieux d'interface entre ville et nature, les dynamiques urbaines associées aux nouvelles réglementations du PnCal, peuvent susciter chez certains, notamment parmi les résidents de longue date, le sentiment qu'ils passent d'un état d'abandon, anciennement lié à l'éloignement et au déficit d'équipement de leur quartier, à celui de dépossession lié à la restriction des usages et à la transformation de leur cadre de vie.

\section{Conclusion}

Historiquement, la création d'espaces naturels protégés est une réponse aux maux urbains basée sur un dualisme spatialisé opposant artifice et nature, production et récréation, grégarité et isolement, aliénation et ressourcement. Cependant, en devenant périurbain, un espace naturel protégé peut-il véritablement garder ce statut historique d'anti-ville? Les ambitions de faire des calanques le premier parc national périurbain d'Europe se sont rapidement heurtées à un tel paradoxe.

Ce lieu est historiquement marqué par une porosité entre ville et nature à laquelle les politiques de requalification environnementale tentent de répondre en se dotant d'une volonté de requalification des marges urbaines. L'effort environnemental demandé aux usagers sur le territoire du Pncal est de ce fait associé à une demande d'effort urbain au sein des quartiers riverains. L'effort environnemental est supporté par les usagers, affectant leurs modes de fréquentation du territoire du PnCal, tandis que l'effort urbain est supporté par les riverains des franges urbaines affectant leur mode d'habiter. Et lorsqu'un riverain est aussi usager du PnCal, ce dernier se voit cumuler effort environnemental et urbain, situation tendant à renforcer l'expression de sentiments d'injustice et de dépossession.

Raisonner avec ces deux notions en parallèle amène à poser la question des lieux où les inégalités environnementales s'exercent. Et c'est peut- 
être autant, voire plus, dans les zones urbaines limitrophes au parc qu'à l'intérieur de celui-ci, que les processus d'étanchéité pèsent sur des pratiques populaires qui avaient su jouer de la grande porosité entre espace urbain et espace naturel. En effet, à travers de nouveaux découpages fonciers, qui s'accompagnent quasi systématiquement de fermetures résidentielles, ce sont des possibilités d'accès à l'espace naturel qui se restreignent pour les riverains les plus modestes.

Enfin, les processus participatifs prônés par le nouveau cadre juridique relatif à la création de parcs nationaux se heurtent aux classiques limites de ce principe démocratique qualifié d'adhocratie par les auteurs les plus critiques (Blanc, 1988; Blatrix, 2000 ; Claeys-Mekdade, 2006) soulignant le caractère ad hoc de ces assemblées et questionnant leur légitimité/représentativité. En lieu et place de la coconstruction d'un consensus, la concertation a servi un arbitrage partial au cœur de conflits préexistants. La configuration du PnCal à laquelle cette concertation a abouti procède d'une institutionnalisation des rapports de force inhérents à ces conflits, légitimant les inégalités environnementales dont ils sont porteurs.

Ces analyses d'un processus en cours doivent être mises à l'épreuve du temps. Si la trajectoire institutionnelle actuelle s'oriente majoritairement vers des formes de sélections socioéconomiques en défaveur des classes populaires urbaines, il convient de prendre au sérieux les formes de résistance passive de ces dernières et leur déclinaison marseillaise faisant de la déviance ordinaire un art de vivre.

\section{Bibliographie}

BlanC M., 1988. Concertation, sociologie urbaine, citoyenneté, Les annales de la recherche urbaine, $\mathrm{n}^{\circ} 38$, juin-juillet, p. 104-111.

Blatrix C., 2000. La «démocratie participative » de Mai 68 aux mobilisations anti-TGV - processus de consolidation d'institutions sociales émergentes, thèse de doctorat en science politique, Université Paris I, 634 p.

Bullard R.D., 2001. Environmental Justice in the $21^{\text {st }}$ Century: Race Still Matters, Philon, no 3/4, vol. 49, p. 151-171.

Cadoret A., 2006. Conflits d'usage liés à l'environnement et réseaux sociaux : enjeux d'une gestion intégrée? Le cas du littoral du Languedoc-Roussillon, thèse de doctorat en géographie-aménagement, Université Paul-Valéry, Montpellier III, $586 \mathrm{p}$.
Charlier B., 1999. La défense de l'environnement, entre espace et territoire : géographie des conflits environnementaux déclenchés en France depuis 1974, thèse de doctorat en géographie, Université de Pau et des Pays de l'Adour, 753 p.

Chateauraynaud F., 2011 . Argumenter dans un champ de forces. Essai de balistique sociologique, Editions Pétra, Paris, 484 p.

Claeys-Merdade C., 2006. La participation environnementale à la française : le citoyen, l'État... et le sociologue, VertigO - la revue électronique en sciences de l'environnement [En ligne], $\mathrm{n}^{\circ} 3$, vol. 7, décembre 2006, mis en ligne le 15 décembre 2006, consulté le 15 décembre 2015. URL : [http://vertigo.revues.org/8446] DOI 10.4000/vertigo.8446.

Claeys-Mekdade C., 2000, Les conflits d'aménagement. Rapports à la «nature » et rapports sociaux. La Camargue, un cas révélateur, thèse de doctorat en sociologie, Université de Provence, Aix-en-Provence, 405 p.

Claeys C., 2014. Créer un parc national des Calanques: préserver, partager ou confisquer un patrimoine socio-naturel?, Annales de Géographie, no 698, vol. 4, p. 995-1015. DOI 10.3917/ag.698.0995.

Claeys C., Gonsales J.N., Barthélémy C., 2012. Marseille et ses natures : perméabilités spatiales, segmentations sociales, Desenvolvimento e Meio Ambiente, jul./dez, vol. 26, p. 69-85.

Daumalin X., 2013 Désindustrialisation et ré-industrialisation à Marseille, fin $\mathrm{XIX}^{\mathrm{e}}$-début $\mathrm{Xx}^{\mathrm{e}}$ siècle. L'exemple des activités chimiques traditionnelles, Rives méditerranéennes, $\mathrm{n}^{\circ} 46$, p. 47-62.

Deldreve V., Candau J., 2014. Produire des inégalités environnementales justes?, Sociologie, n 3, Vol. 5, p. 255-269. DOI 10.3917/socio.053.0255.

Deldreve V., Deboubt Ph. (dir.), 2012. Le Parc national des Calanques - Construction territoriale, concertation et usages, Versailles, Quae, 229 p.

Deldreve V., Hérat A., 2012. Des inégalités garantes de la protection des Calanques?, VertigO - la revue électronique en sciences de l'environnement [En ligne], $\mathrm{n}^{\circ} 2$, vol. 12, septembre 2012, mis en ligne le 31 octobre 2012, consulté le 15 décembre 2015 [http://vertigo.revues.org/12700. DOI 10.4000/vertigo. 12700].

Deldrève V., 2011 . Préservation de l'environnement littoral et inégalités écologiques. L'exemple du Touquet-Paris Plage. Espaces et Sociétés, no 144-145, p. 173-187.

Donzel A., 1998. Marseille, l'expérience de la cité, Paris, Anthropos, $196 \mathrm{p}$.

Donzel A., 2014, Le nowvel esprit de Marseille, Paris, L'Harmattan, $176 \mathrm{p}$.

Ginelli L., Marquet V., Deldreve V., 2014. Bien pratiquer la nature... pour protéger les Calanques?, Ethnologie française, $\mathrm{n}^{\circ} 3$, vol. 44, p. 525-536. DOI 10.3917/ ethn. 143.0525

Godard O., 2004. Autour des conflits à dimension environnementale. Évaluation économique et coordination dans un monde complexe, Cahiers d'économie Politique/Papers in Political Economy, n 47, vol. 2, p. 127-153.

Gontien C., 1991. Le cabanon marseillais. Images et pratiques, Marseille, Cerfise, 299 p. 
Guyot S., Dellier J., Cerbelaud F., 2014. L'environnement au profit des plus riches? Construction et hybridation d'un front écologique métropolitain dans la Péninsule du Cap (Afrique du sud), Vertigo, $\mathrm{n}^{\circ}$ 1, vol. 14 [http://vertigo.revues. org/14660] DOI 10.4000/vertigo. 14660.

HÉRAT A., 2012. Une politique de nature à l'épreuve du territoire : le quartier de la Cayolle et la calanque de Sormiou (Marseille), in Deldrève V., Deboudt Ph. (dir.), Le Parc national des Calanques - Construction territoriale, concertation et usages, Versailles, Quae, p. 53-72.

FABurel G., 2010. Débats sur les inégalités environnementales. Une autre approche de l'environnement urbain, justice spatiale / spatial justice, 2, p. 102-132 [http://www.jssj.org/ archives/02/medialdossier_focus_vo5.pdf].

Holifield R., 2001. Defining Environmental Justice and Environmental Racism, Urban Geography, n 1, vol. 22, p. 78-90.

Laigle L., 2005. Les Inégalités écologiques de la ville. Caractérisation des situations et de l'action publique. Programme MÉTATTM-MEDD, Recherche pour le PUCAMÉTATTM, Rapport Intermédiaire, 1, Centre Scientifique et Technique du Bâtiment, Paris, 122 p. (non publié).

Larrère R., Lizet B., Berlan-Darqué M. (dir.), 2009. Histoire des parcs nationaux. Comment prendre soin de la nature, Paris, Quae, 240 p.

Laslaz L. (dir.), 2015. Les espaces protégés. Entre conflits et acceptation, Paris, Belin, 432 p.

Lipietz A., 1998. Économie politique des écotaxes. Rapport au Conseil d'Analyse Économique du Premier ministre, Conseil d'Analyse Économique n ${ }^{\circ}$ 8, Paris, La Documentation Française, $81 \mathrm{p}$.
Martinez-Alier J., 2008. Conflits écologiques et langage de valorisation, Écologie et politique, $\mathrm{n}^{\circ}$ 35, vol. 1, p. 91-107. DOI 10.3917/ecopo.035.0091.

Massena-Gourc G., 1994. La protection à l'épreuve de la diversité des usages, le massif des Calanques, Études Rurales, $n^{\circ} 133-134$, p. 149-162.

Mormont M., 2006. Conflit et territorialisation, Géographie, économie, société, no 3, vol. 8, p. 299-318.

Péraldi M., Duport C., Samson M., 2015. Sociologie de Marseille, Paris, La découverte, 128 p.

Pye S., Skinner I., Meyer-Ohlendorf N., Leipprand A., Lucas K., Salmons R., 2008. Addressing the social dimensions of environmental policy - A study on the linkages between environmental and social sustainability in Europe, European Commission Directorate-General/Employment, Social Affairs and Equal Opportunities, 50 p. (non publié)

Roncayolo M., 1996. Les Grammaires d'une ville. Essai sur la genèse des structures urbaines à Marseille, Paris, Éditions de l'EHESS, 507 p.

TAtoni T. (dir.), 2013. Caractérisation de la fréquentation littorale et détermination d'indicateurs de vulnérabilité écologique pour définir des modes de gestion durable - Cas de la bande littorale du projet de parc national des Calanques, Rapport final, APR LITEAU, MEDDT, 131 p. (non publié).

TAYLOR D.E., 2000, The Rise of Environmental Justice Paradigm. Injustice framing and the Social Construction of Environmental Discourses, American Behavioural Scientist, $n^{\circ} 4$, vol. 43, p. 508-580.

Témime É., Deguigné N., 2001. Le camp du Grand Arénas Marseille, 1944-1966, Paris, Autrement, 157 p. 